\section{Trauma e sintomas de estresse pós-traumático em dependentes químicos*}

Prezados Editores,

Estima-se que a prevalência de transtorno de estresse pós-traumático (TEPT) na população varie de 1 a 14\% ${ }^{1}$, podendo alcançar índices de 60 a $80 \%$ em populações traumatizadas $^{2}$. As investigações com grupos específicos de pessoas que procuram tratamento têm encontrado que 30 a 50\% de usuários de substâncias psicoativas são portadores de TEPT $^{3}$.

A exposição ao trauma é a condição sine qua non para o desenvolvimento do TEPT, e um estudo epidemiológico americano encontrou que cerca de $90 \%$ da população tinha sido exposta a algum evento traumático ${ }^{4}$. Pelos comportamentos de risco, os dependentes químicos configuram-se como um grupo de grande vulnerabilidade para traumas e TEPT.

A condição comórbida entre uso de substâncias psicoativas e TEPT tem sido, ainda, pouco estudada em nosso meio, e nos propomos a investigar a ocorrência de traumas psicossociais e presença de sintomas de TEPT em uma amostra de dependentes químicos. Foram avaliados, na primeira consulta, 23 pacientes, com, no mínimo, 1 mês de abstinência, que procuraram o Instituto de Prevenção e Pesquisa em Álcool e outras Dependências (IPPAD) para tratamento, no período de março a julho de 2006. Eles responderam à Specific Traumatic Events ${ }^{4}$, lista de eventos, desenvolvida em acordo com os critérios do Manual de Diagnóstico e Estatística das Perturbações Mentais (DSM-IV) e Davidson

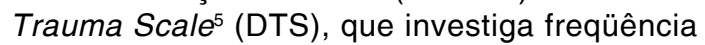
e severidade dos sintomas de TEPT.

Os pacientes são do sexo masculino, com idades entre 18 e 30 anos $(39,1 \%)$, ensino fundamental incompleto $(30,4 \%)$, e utilizam apenas álcool (13\%). Todos apresentam dependência grave da substância psicoativa e internações hospitalares anteriores, sendo que $38,1 \%$ tiveram três ou mais hospitalizações.

* Pesquisa realizada no Instituto de Prevenção e Pesquisa em Álcool e Outras Dependências (IPPAD) e aprovada pelo Comitê de Ética em Pesquisa da Pontifícia Universidade Católica do Rio Grande do Sul (PUCRS), Porto Alegre, RS (CEP 06/03028 em 13/03/06).
Todos os pacientes (100\%) estiveram expostos a algum evento traumático durante sua vida. O evento mais prevalente foi ter sido "assaltado ou ameaçado com arma", relatado por $56,5 \%$ dos dependentes químicos. A "morte súbita e inesperada de algum parente ou amigo próximo" foi vivenciada por $54,5 \%$ da amostra, tendo sido relatada como o "pior" evento por $28,6 \%$ dos respondentes. Estudos epidemiológicos têm mostrado a alta prevalência desse trauma na população, com um risco moderado para TEPT $^{4}$.

Algum sintoma de TEPT foi relatado por todos os participantes do estudo, e $47,6 \%$ tiveram "imagens, memórias ou pensamentos dolorosos sobre o evento" na última semana, com 9,5\% experimentando diariamente esse sintoma. Do total de pacientes, $24 \%$ sentiramse perturbados de forma moderada a extrema. Mais da metade (57\%) relatou "dificuldades para se sentir satisfeito com as coisas de sua vida". Destes, $19 \%$ sentiram-se muito perturbados, e $14,3 \%$ classificaram sua perturbação como extrema.

Esse sintoma pode confundir-se com o de transtorno depressivo, que ocorre em altas prevalências, tanto em traumatizados que não desenvolveram TEPT como em portadores de TEPT e dependentes químicos. Daí a importância de uma investigação comórbida criteriosa e atenção aos traumas em dependentes químicos como prevenção para o desenvolvimento de TEPT.

\section{REFERÊNCIAS}

1. American Psychiatric Association. DSM-IV: manual diagnóstico e estatístico de transtornos mentais. 4.ed. Porto Alegre; Artmed; 1995.

2. Kapczinski F, Margis R. Transtorno de estresse póstraumático: critérios diagnósticos. Rev Bras Psiquiatr. 2003;25(Supl 1):3-7.

3. Brady KT, Randall CL. Gender differences in substance use disorders. Psychiatr Clin North Am. 1999;22(2):24152.

4. Breslau N, Kessler RC, Chilcoat HD, Schultz LR, Davis GC, Andreski P. Trauma and posttraumatic stress disorder in the community. Arch Gen Psychiatry. 1998;55(7):626-32.

5. Davidson JR, Book SW, Colket JT, Tupler LA, Roth S, David D, et al. Assessment of a new self-rating scale for post-traumatic stress disorder. Psychol Med. 1997;27(1):153-60.

Title: Trauma and posttraumatic stress disorder symptoms in drug users 
Sibele Faller

Psicóloga, Instituto de Prevenção e Pesquisa em

Álcool e Outras Dependências (IPPAD) e Centro de

Pesquisa em Álcool e Drogas (CEPAD), Universidade

Federal do Rio Grande do Sul (UFRGS), Porto Alegre, RS.

Gilda Pulcherio

Psiquiatra, IPPAD, Porto Alegre, RS. Mestre em

Psicologia Social e da Personalidade, Pontifícia

Universidade Católica do Rio Grande do Sul

(PUCRS), Porto Alegre, RS.
Marlene Strey

Psicóloga, IPPAD, Porto Alegre, RS. Professora, Programa de Pós-Graduação em Psicologia, PUCRS, Porto Alegre, RS.

Correspondência:

Gilda Pulcherio

Rua Dona Lida Monteiro, 450, Bairro São Caetano CEP 91720-300 - Porto Alegre, RS

E-mail: gilda @ ippad.com.br

Copyright (C) Revista de Psiquiatria

do Rio Grande do Sul - SPRS 